\title{
Assessment of Laser Joining Quality by Visual Inspection, Computer Simulation, and Deep Learning
}

\author{
Chang Min Han ${ }^{1}$, Tae Won Kim $^{1}$ and Hae Woon Choi ${ }^{2, *(D)}$ \\ 1 Graduate School of Mechanical Engineering, Keimyung University, 1095 Dalgubeol Daero, \\ Daegu 42601, Korea; ssunchib22@naver.com (C.M.H.); xodnjs813@naver.com (T.W.K.) \\ 2 Department of Mechanical Engineering, Keimyung University, 1095 Dalgubeol Daero, Daegu 42601, Korea \\ * Correspondence: hwchoi@kmu.ac.kr; Tel.: +82-53-580-5216
}

check for updates

Citation: Han, C.M.; Kim, T.W.; Choi, H.W. Assessment of Laser Joining Quality by Visual Inspection, Computer Simulation, and Deep Learning. Appl. Sci. 2021, 11, 642. https://doi.org/10.3390/app11020642

Received: 23 December 2020

Accepted: 7 January 2021

Published: 11 January 2021

Publisher's Note: MDPI stays neutral with regard to jurisdictional clai$\mathrm{ms}$ in published maps and institutional affiliations.

Copyright: () 2021 by the authors. Licensee MDPI, Basel, Switzerland. This article is an open access article distributed under the terms and conditions of the Creative Commons Attribution (CC BY) license (https:// creativecommons.org/licenses/by/ $4.0 /)$.
Featured Application: Polymer joining is an important technology in automotive and industrial applications. Lasers are an attractive heat source for polymer joining because they are more controllable, productive, and easy to automate and cause minimal collateral damage to heat-affected zones. However, the assessment of quality evaluation remains a problem for reliable products in mass production. Laser polymer joining requires a non-destructive evaluation, and recent developments in deep learning have begun a new era for this requirement. We believe that deep learning technology can be used to provide state-of-the-art polymers that can improve quality assessment in the non-destructive polymer joining process.

Abstract: Polymer joining results are evaluated and compared in different ways, such as visual inspection, computer simulation, and deep learning analysis, to assess the joining quality. For the experiments, energies in the range of 3 to $5 \mathrm{~J} / \mathrm{mm}$ were used from preliminary experimental data. A total of 15 welding experiment schedules were performed. Weld defects due to a lack of fusion were detected in some regions of specimens treated with a low-power laser region $(3 \mathrm{~J} / \mathrm{mm})$, where a lack of fusion, in turn, occurred due to underheating. Bubble-shaped weld defects were observed in some specimens treated with a high-power laser region $(5 \mathrm{~J} / \mathrm{mm})$; melting occurred due to the overheating of the specimen. Computer simulations were used to trace the boundaries of the fusion zone, and yielded results similar to the visual inspection ones. In the lower-energy region, the energy may not be sufficient to fuse the specimen, whereas the high-energy region may have sufficient energy to break down the polymer chains. A novel deep learning algorithm was used to statistically evaluate the weld quality. Approximately 1700-1900 samples were collected for each condition, and the pre-trained quality evaluation indicated a highly reliable $(>98 \%)$ welding classification (fail or good). According to the results of this study, welding quality assessments based on visual inspection, computer simulation, and DL-based inspection yield similar results.

Keywords: laser welding; deep learning; weld quality assessment; computer simulation; polymer joining

\section{Introduction}

Polymer materials are widely used in the interiors and exteriors of automobiles because they are lighter than metallic materials. Their excellent strength and moldability at low temperatures represent additional advantages of polymers, as this reduces production costs and enhances design flexibility [1,2]. Researchers have developed various techniques for producing components from polymer materials. Adhesive and mechanical fastening are typical bonding methods for polymers, but the recent development of direct thermal energy for joining has reduced costs and provided eco-friendly products. Various joint configurations were compared to distinguish the bonding quality, and the use of chromium interlayers allowed an increase in adhesion forces. Laser beams, ultrasonic waves, and 
radio frequency $(\mathrm{RF})$ are also commonly used for polymer joining. Joining techniques have improved significantly over the past few decades [1-6].

A laser is a very versatile, controllable, and easy to use tool for the polymer joining process. In particular, direct thermal energy deposition provides strong joins, and there is little possibility of contamination during the joining process [2,5]. Furthermore, joining using thermal energy can be easily applied to various thermoplastic polymeric materials during the fabrication of automotive parts, biomedical devices, and electrical devices [2-5]. The advantages of direct joining include lower costs compared to adhesives and the ecofriendly nature of the technology, as no chemicals are used.

Lasers are also a preferred thermal energy source, as they are readily available in the industry, easy to use, and highly precise [3-6]. Lasers are also less sensitive to materials and can be used for most types of polymer joining, such as joining polymers to other polymers, glass, or other materials. They also have the advantage of good sealing, which produces better esthetic results after joining compared to other joining processes, such as ultrasonic and adhesive joining [2]. In laser joining, the thermal energy imparted by the laser beam is absorbed in the interface between transparent and opaque materials. The top layer is transparent to the laser, while the bottom layer is opaque [2,7]. As the most absorptive wavelength can be used for laser joining, the joining efficiency can be maximized without damaging the surrounding area. After the energy has been absorbed on the boundary, the joining region is heated to over the glass transition temperature and forged by an external pressing force $[8,9]$.

In the era of the 4th industrial revolution, artificial intelligence (AI) technology using deep learning (DL) is being applied in various fields [10-18]. A computer vision-based DL algorithm has been used for autonomous vehicle driving, games, and medical applications. Recently, this technology has also been used to improve the production quality in manufacturing applications [11-14]. In DL for product quality monitoring, the greater the amount of correctly classified training data, the more accurate the results are.

A system for monitoring porosity in laser welding processes was developed using a DL algorithm [10], and real-time weld pool data were collected. DL was also used to predict the hardness distribution with the laser heat treatment of AISI H13 tool steel [11]. During the fabrication of sensor systems using laser welding processes, DL-based computer vision systems have been used to capture signals indicating the welding status [10]. A monitoring system to determine the degree of penetration during laser welding has also been introduced [10].

Convolution neural networks (CNNs) are the most commonly used networks for image-based DL. CNNs, as multilayer perceptrons, have layers in which each neuron is connected to all the neurons in the next layer. CNNs analyze hierarchical patterns in data and then generate smaller, simpler patterns. These networks also employ encoderdecoder structures to preserve the prominent features of images [15]. The successful use of CNN-based DL algorithms for product quality monitoring has been reported by many researchers.

This paper reports the results of a comparative analysis of the joining of polycarbonate (PC) and acrylonitrile butadiene styrene (ABS) polymeric materials with different characteristics using a diode laser. The weld quality was evaluated by (1) visual inspections, (2) computer simulations, and (3) DL techniques, and the results were then compared.

\section{Experiments}

\subsection{Experimental Setup}

A diode laser was used as the heat source in the experiments because these lasers are widely used for polymer bonding due to their excellent efficiency and ease of control. As shown in Figure 1, a laser beam resonated by the diode laser (c) was transmitted through fiber optics. The specimen was placed on the working table controlled by a computer numerical control $(\mathrm{CNC})$ machine (a), and integrated control was achieved using a laptop 
computer (b). The delivered laser beam was focused on the specimen through a focusing lens installed at the head of the CNC.
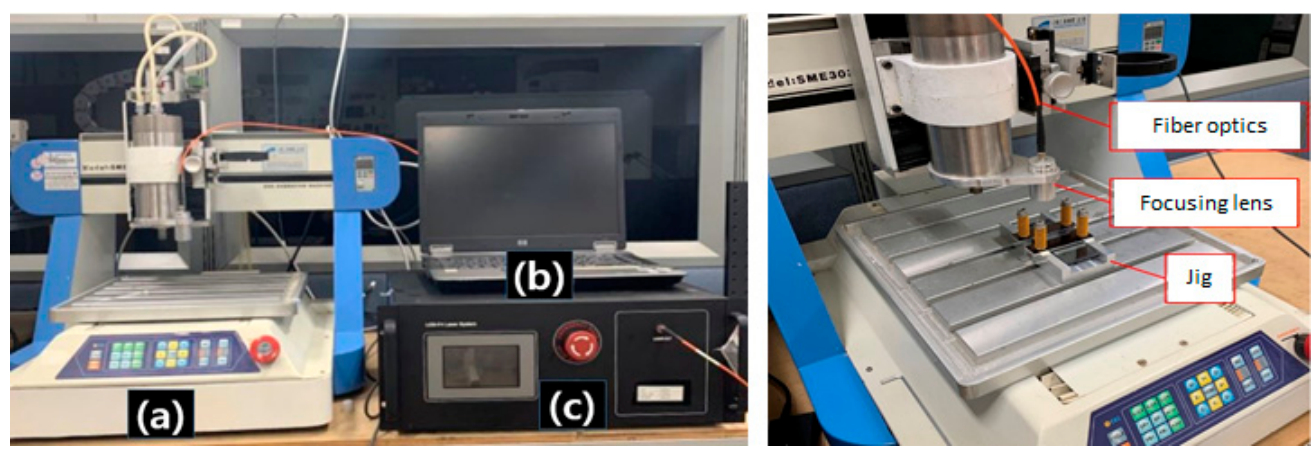

Figure 1. Experimental setup.

The laser beam was transferred to the CNC head, and the distance between the workpiece and focus lens $(\mathrm{FL}=50 \mathrm{~mm})$ could be adjusted to obtain the desired beam size. In the experiments, the focused spot diameter was set to $5 \mathrm{~mm}$ based on the preliminary experimental results. A 3-axis-controlled CNC machine (SME3030; SME Trading) was used for the precise transfer of specimens by G-code, and both the laser and specimen transfers were controlled using the laptop. The laser generated a continuous waveform (CW) with a wavelength of $980 \mathrm{~nm}$, and the output was set in the range of 3 17 Watts. The welding speed was set in the range of $60 \sim 250 \mathrm{~mm} / \mathrm{min}$ depending on the output power. A total of 15 combinations of laser power and welding speed were used to achieve an input energy per unit length of $3 \sim 5 \mathrm{~J} / \mathrm{mm}$; the detailed welding schedules are summarized in Table 1 .

Table 1. Matrix of welding schedule.

\begin{tabular}{|c|c|c|c|}
\hline \multicolumn{2}{|c|}{ Input Energy (J/mm) } & \multirow{2}{*}{$\frac{\text { Power (Watt) }}{3}$} & \multirow{2}{*}{$\frac{\text { Welding Speed }(\mathrm{mm} / \mathrm{min})}{60}$} \\
\hline$\# 1-1$ & 3 & & \\
\hline$\# 1-2$ & 3 & 5.5 & 110 \\
\hline$\# 1-3$ & 3 & 8 & 160 \\
\hline$\# 1-4$ & 3 & 10.5 & 210 \\
\hline$\# 1-5$ & 3 & 13 & 250 \\
\hline \#2-1 & 4 & 5 & 75 \\
\hline$\# 2-2$ & 4 & 7.5 & 112.5 \\
\hline \#2-3 & 4 & 10 & 150 \\
\hline \#2-4 & 4 & 12.5 & 187.5 \\
\hline$\# 2-5$ & 4 & 15 & 225 \\
\hline$\# 3-1$ & 5 & 7 & 84 \\
\hline$\# 3-2$ & 5 & 9.5 & 114 \\
\hline$\# 3-3$ & 5 & 12 & 144 \\
\hline$\# 3-4$ & 5 & 14.5 & 174 \\
\hline$\# 3-5$ & 5 & 17 & 204 \\
\hline
\end{tabular}

The irradiated laser beam passed through the transparent PC material and was absorbed on the boundary by the ABS material (through transmission). The absorbing layer was heated to the glass transition temperature, which is approximately 135 and $110{ }^{\circ} \mathrm{C}$ for PC and ABS, respectively [19]. Then, the absorbed heat energy on ABS transferred to the contact PC layer, which resulted in a crosslinking of polymer chains at the contact boundary. 
For the experiments, the specimens on the joining jig were prepared, and the materials were cut to dimensions of $42 \times 100 \times 6.5 \mathrm{~mm}$ for ABS and $42 \times 100 \times 3 \mathrm{~mm}$ for PC. The specially designed jig shown in Figure 2 was used to hold the weld specimens. Precision mold springs and wrench bolts were used to maintain a precise and constant pressing force on the specimens via pressure plates made of aluminum. The mold springs had an inner diameter of $6 \mathrm{~mm}$, an outer diameter of $12 \mathrm{~mm}$, a free length of $40 \mathrm{~mm}$, and a spring constant of $0.7 \mathrm{~kg}_{\mathrm{f}} / \mathrm{mm}$. The wrench bolts were tightened in four places to yield a force of $22.4 \mathrm{~kg}_{\mathrm{f}}$ in total.
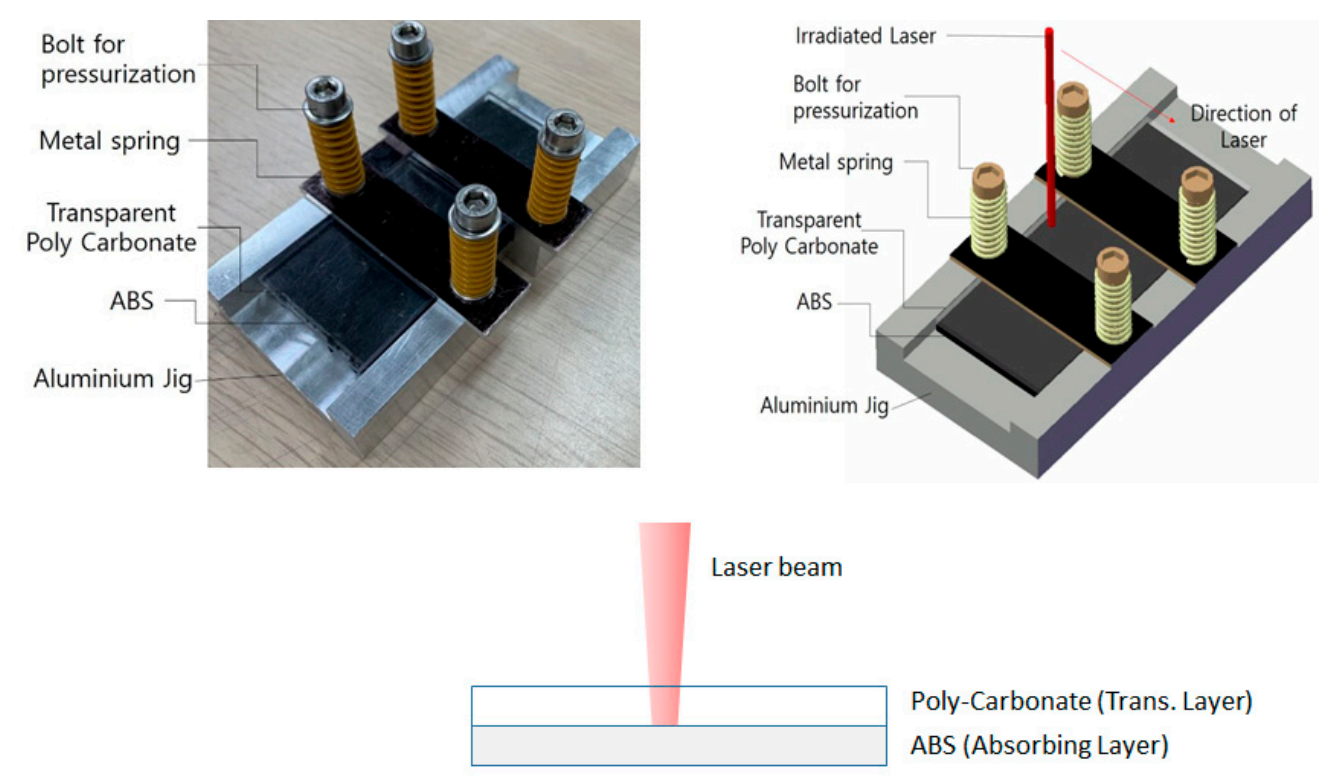

Figure 2. Welding jig configuration.

\subsection{Computer Simulation}

A computer simulation can be effectively used to track the fusion boundary based on the temperature distribution. By calculating the temperature distribution, the weld quality can be estimated indirectly. The heat transfer module of the COMSOL Multiphysics software (ver. 5.3a; COMSOL Inc.) was used and the results were validated based on prior research, where the simulated temperature distribution was compared to the actual temperatures measured by thermocouples and infrared (IR) cameras.

For the simulation, a blocked domain of dimensions $20 \times 10 \times 2 \mathrm{~mm}$ was used, and a linear heating source with a Gaussian-shaped laser beam was assumed to be as described in Equation (1).

$$
Q(x, y, z)=Q_{0}\left(1-R_{c}\right) \cdot \frac{A_{c}}{\pi \sigma_{x} \sigma_{y}} e^{-\left[\frac{\left(x-x_{0}\right)^{2}}{2 \sigma_{x}{ }^{2}}+\frac{\left(y-y_{0}\right)^{2}}{\left.2 \sigma_{y}\right)^{2}}\right]} \cdot e^{-A_{c} z},
$$

where $Q_{0}$ is the peak power, $R_{c}$ is the reflectance, $\sigma_{x}$ and $\sigma_{y}$ are the beam waists in the $x$ and $y$ directions, and $A_{c}$ is the skin depth/absorption layer thickness.

As described in Equation (1), the irradiating laser beam was assumed as a $2 \mathrm{D}$ ellipsoidal distribution, with $A_{\mathcal{c}}$ of skin depth with respect to the z-direction. The skin depth can be analytically calculated for the metallic material, but it was found from computer simulation in this research. The reflectance $R_{c}$ value is also an important value, and it was found by the experimental data analysis by using an integrating sphere.

To trace the temperature distribution, multiple probe points were assigned to various locations. The simulation conditions and schedules were identical to the experimental ones 
which are described in Table 1. As the governing equation for the simulation domain, a general 3D heat transfer equation was used, as described as Equation (2).

$$
\begin{gathered}
\rho C_{p}\left(\frac{\partial T}{\partial t}+\mathbf{u}_{\text {trans }} \cdot \nabla T\right)+\nabla \cdot\left(q+q_{r}\right)=-\alpha \mathrm{T}: \frac{d S}{d t}+Q, \\
q=-k \nabla T \text { or } h\left(T_{\text {ext }}-T\right),
\end{gathered}
$$

where Equation (3) is a conductive $(\kappa)$ or convective $(h)$ heat transfer through the surrounding bodies. In Equation (2), $\rho$ is the density of the polymers; $C_{p}$ is the specific heat capacity at constant stress; $T$ is the absolute temperature; $u_{\text {trans }}$ is the velocity vector of translational motion; $q$ and $q_{r}$ are the heat flux caused by conduction and radiation, respectively. $\alpha$ is the coefficient of thermal expansion, $S$ is the second Piola-Kirchhoff stress tensor, and $Q$ contains additional laser heat sources which can be described using Equation (1).

\subsection{Deep Learning Experiment}

Given the importance of reliable training for DL, large amounts of data were collected prior to the DL process. To construct the dataset, a series of images of specimens welded under conditions of 3,4 , and $5 \mathrm{~J} / \mathrm{mm}$ was acquired. A charge-coupled device (CCD) camera (STC-SC33POE; Sentech) with a resolution of $640 \times 480$ was installed and a GigE connection was used for image collection. A linear translation stage (DDSM100/M; Thorlabs) was used for precise specimen translation (Figure 3). The specimens were transferred on a linear translation stage at a rate of $0.8 \mathrm{~mm} / \mathrm{s}$, and image data were acquired every $100 \mathrm{~ms}$ with $10 \mathrm{~ms}$ of exposure time. For each condition, approximately 1700-1900 sets of data were collected, and up to 28,500 images were used in the DL process.
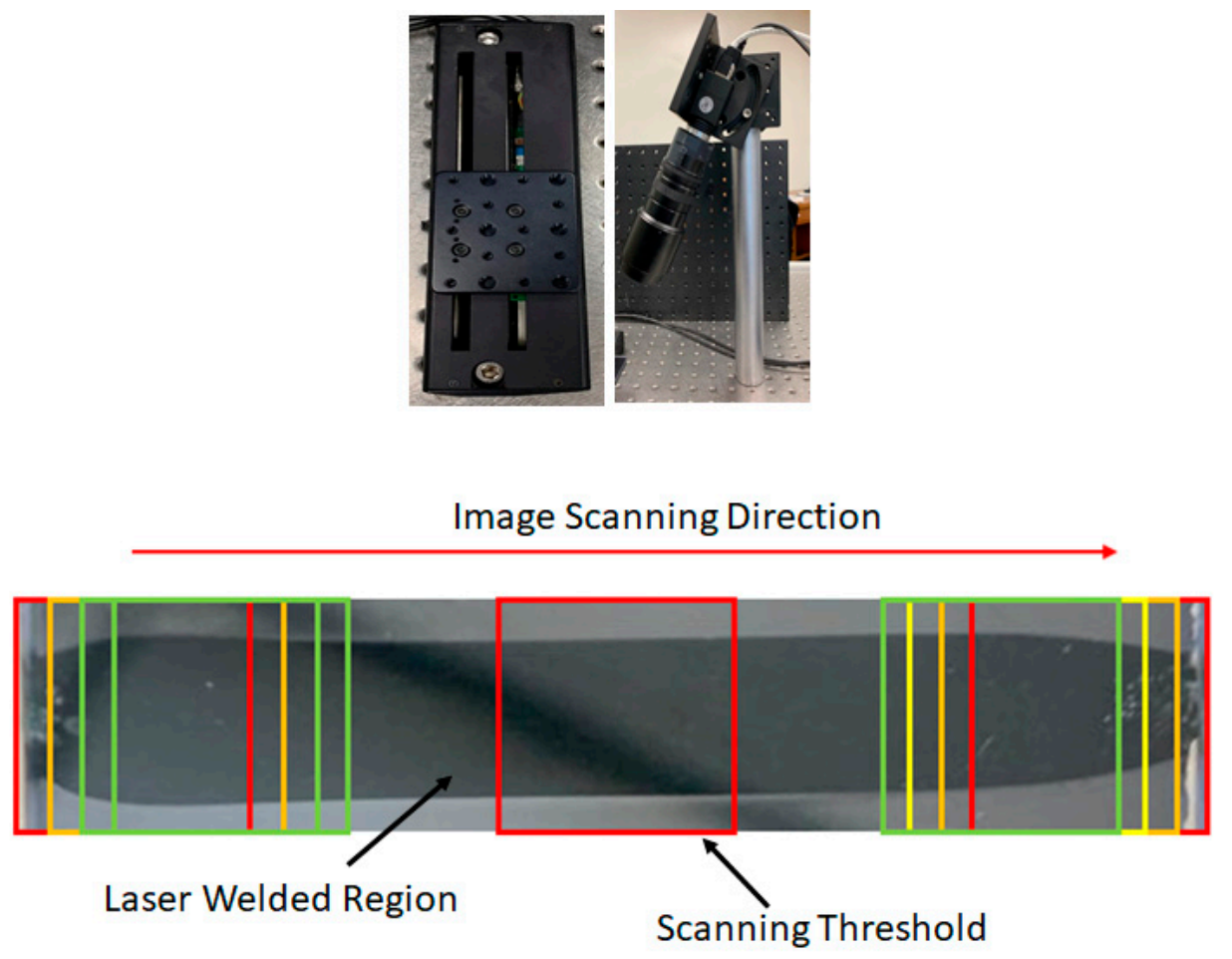

Figure 3. Progressive image acquisition process.

The images collected for each condition were divided into two categories, "Good" and "Fail", for the quality assessment. The acquired images had a resolution of $648 \times 494$, which was re-adjusted to $227 \times 227$ to match the input format of the DL algorithm (AlexNet). As shown in Figure 5, the straightness of the edges in the weld beads was used to assess the weld quality as "Good" or "Fail" by visual inspection. The DL algorithm was implemented 
in MATLAB (ver. 2019b; MathWorks Inc.) using the DL toolbox and an NVIDIA GPU (RTX 2070).

The MATLAB Parallel Computing Toolbox was used for implementation. The parallel computation was performed using CUDA (ver. 10.2; NVIDIA) to improve the speed of the algorithm by exploiting parallelism. The DL-based image processing algorithm was in turn based on an AlexNet CNN. A special program was coded to recognize videos and images, even if the image size or position was randomly placed. The AlexNet CNN consisted of 5 convolutional layers and 3 fully connected layers. For this research, the AlexNet was modified and the details of the DL process structure of DL are described in Figure 4.

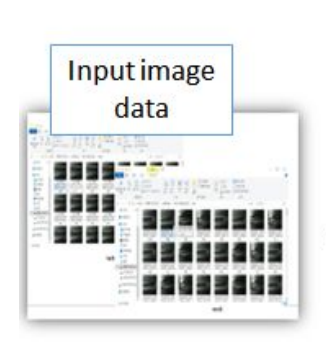

Input data

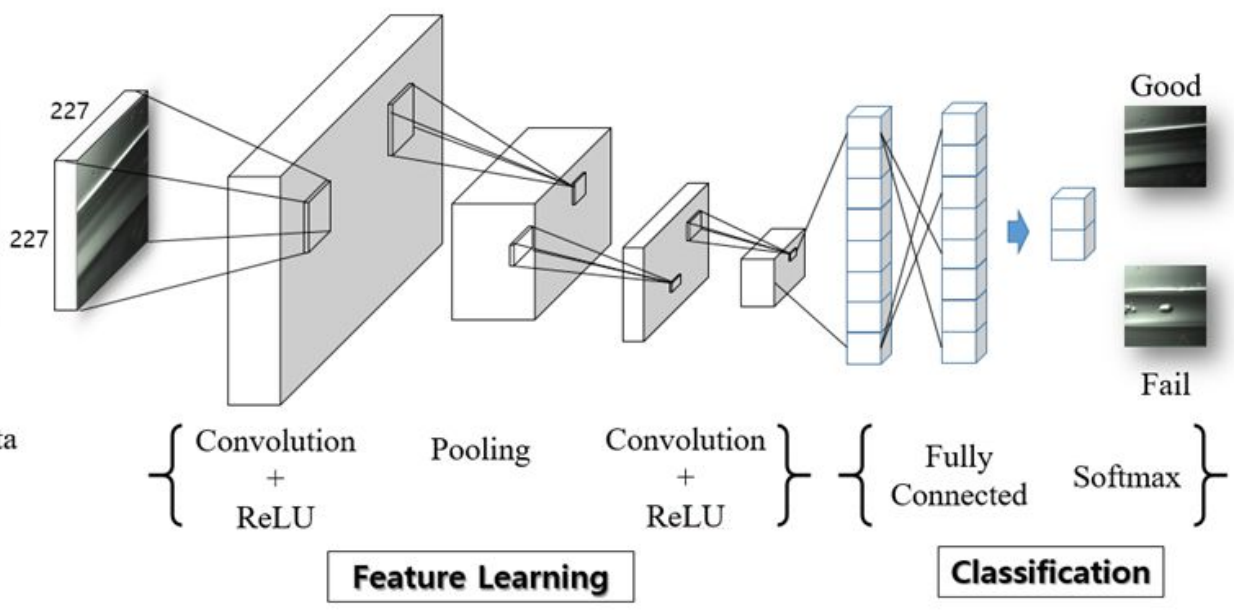

Figure 4. Structure of the deep learning algorithm.

The modified DL architecture consists of 8 layers, which include 5 convolutional layers and 3 fully connected layers. It uses Rectified Linear Units (ReLU) for lower error and faster convergence. The maximum number of repetitions was set at 30 and the mini-batch size was set to 128 from the preliminary DL research. Based on a series of training processes, it took less than a second to evaluate the welding quality when the algorithm was pretrained on 28,500 photographs using a spatial gray-level dependence matrix (SGDM). Overall, the predictive accuracy reached approximately $99 \%$ through repeated learning.

The pretrained network, as described in Figure 4, was adapted to our welding bead data. To apply the developed deep learning algorithm, we created a custom training loop using automatic differentiation. In a commercial deep learning toolbox provided by MATLAB and the initialization process was conducted for the weights and biases, which are given by the internal function-the "WeightsInitializer" and "BiasInitializer" properties of the layers, respectively. To minimize the loss function for the convergence, the standard gradient descent algorithm was used to update the network parameters, such as weights and biases, and basic mathematical equations.

In addition, to expedite the converging time and increase accuracy, the stochastic gradient descent with momentum algorithm was used to evaluate the gradient and update the parameters using a subset of the training welding data. The mini-batch size and the maximum number of epochs were optimized for accuracy. The basic governing equation is described in Equation (4):

$$
\theta_{i+1}=\theta_{i-\alpha} \nabla E\left(\theta_{i}\right)+\gamma\left(\theta_{i}-\theta_{i-1}\right)
$$

where $i$ is the iteration number, $\alpha>0$ is the learning rate, $\theta$ is the parameter vector, $E(\theta)$ is the loss function, and $\gamma$ determines the contribution of the previous gradient step to the current iteration. In the standard gradient descent algorithm, the gradient of the loss function, $\nabla E(\theta)$, is evaluated using the entire training set, and the standard gradient descent algorithm uses the entire data set at once. 


\section{Results}

\subsection{Welding Experiment and Visual Inspection Results}

The experiments were performed based on the welding schedule presented in Table 1, and the results are summarized in Figure 5. The welding was inspected visually for melting, overheating, or a lack of fusion caused by underheating. As described in the previous chapter, the welding experiments were performed with input energies in the range of $3 \sim 5 \mathrm{~J} / \mathrm{mm}$, but in some areas welding failure occurred due to under or overheating.
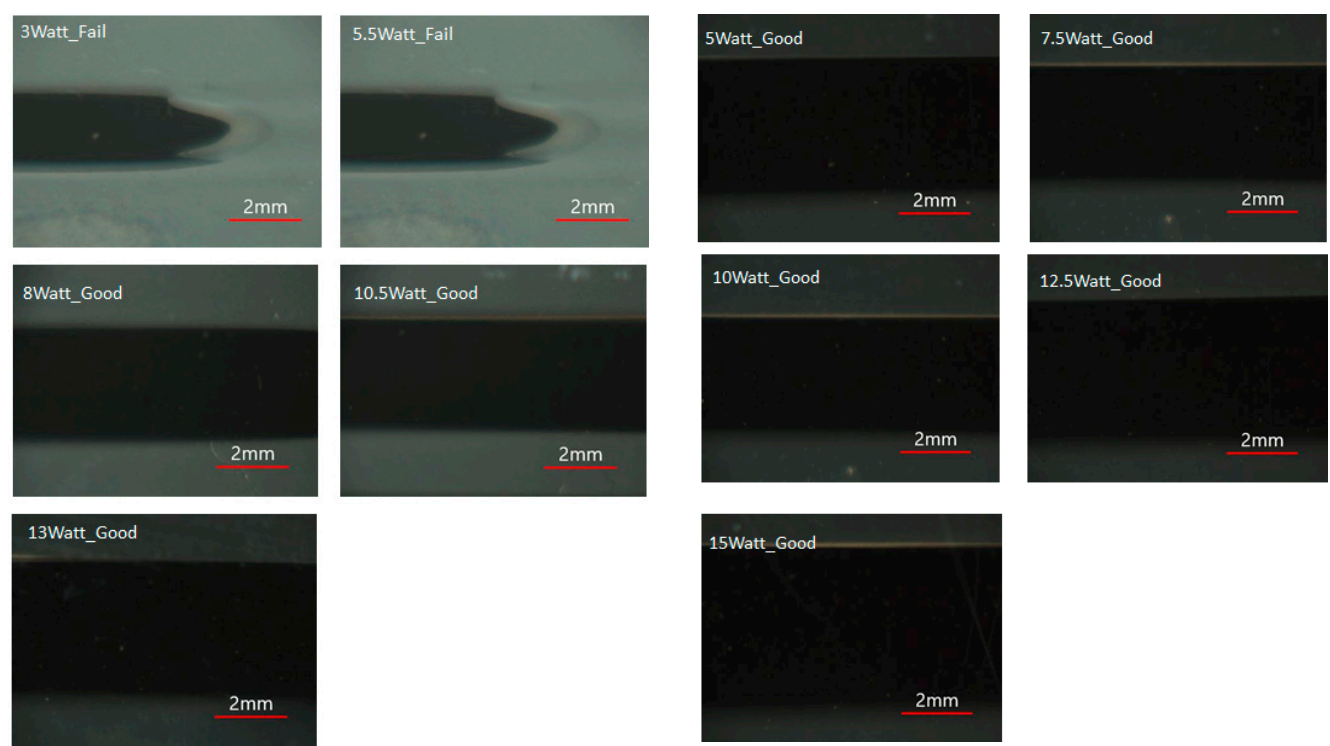

(a) Training data for $3 \mathrm{~J} / \mathrm{mm}$.

(b) Training data for $4 \mathrm{~J} / \mathrm{mm}$.
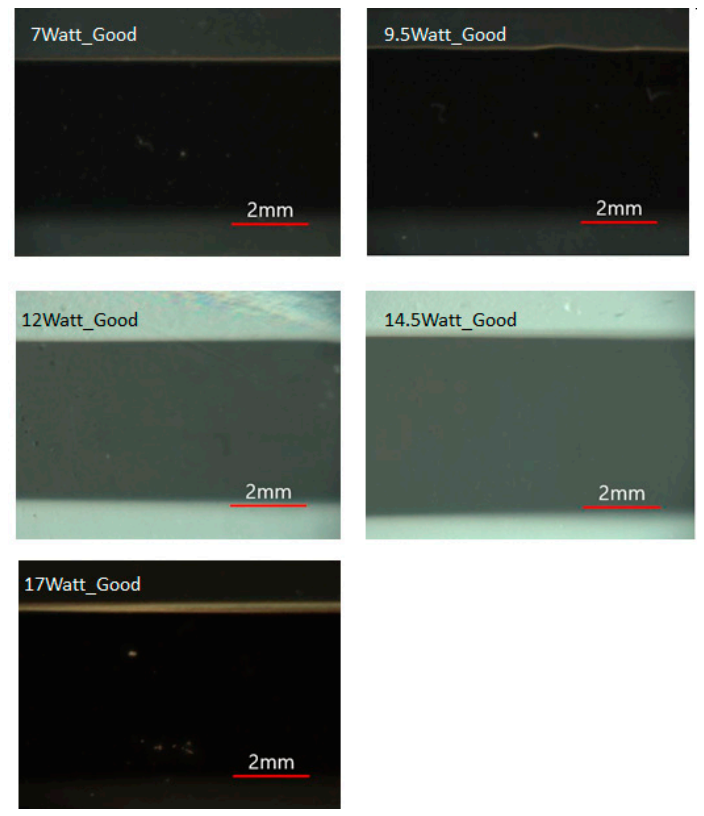

(c) Training data for $5 \mathrm{~J} / \mathrm{mm}$.

Figure 5. Training data sets for different energy inputs.

Typically, excessive heating resulted in bubble defects in the central region, while underheating resulted in a lack of fusion in the edge boundary region. The results of the visual inspections are summarized in Figure 6. As shown in the graph, a lack of fusion (experiment \#1-1) due to underheating was observed under the low-power, low-speed conditions in the $3 \mathrm{~J} / \mathrm{mm}$ region. However, in the $5 \mathrm{~J} / \mathrm{mm}$ area, some overheating occurred under the high-power, high-speed conditions (experiment \#3-5). 


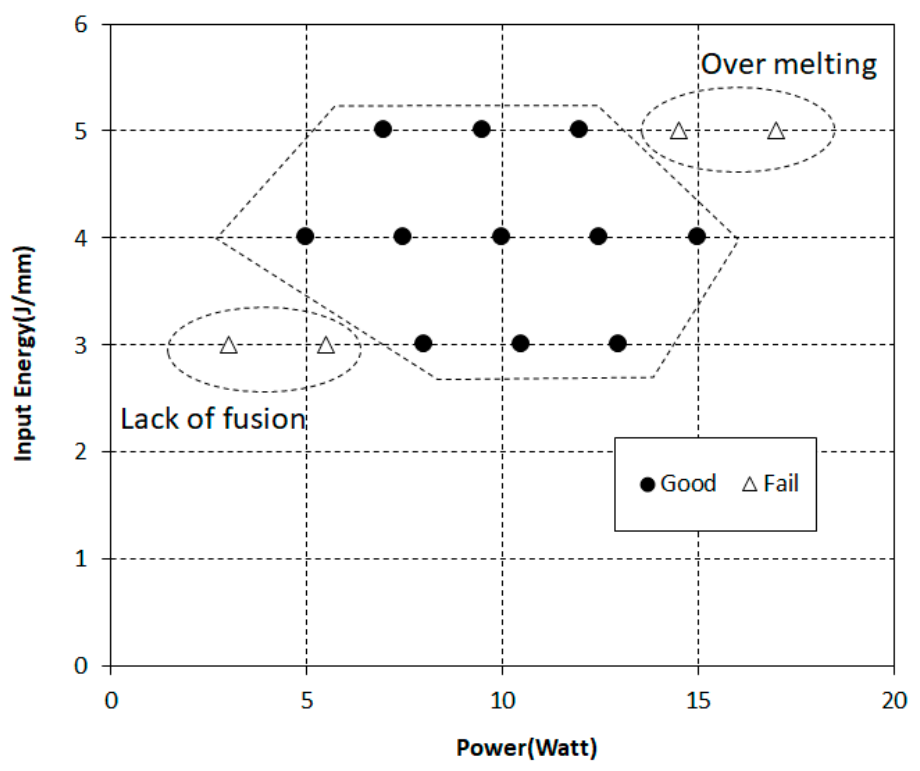

Figure 6. Results of the welding evaluated by visual inspection.

\subsection{Computer Simulation}

For the computer simulation, we followed the procedure used in a previous study. The results of the simulation are summarized in Figure 7. The output and welding speed of the laser beam are described in Table 1. The focused spot diameter was set to $5 \mathrm{~mm}$, which is identical to the actual welding condition, and the absorption of the laser heat source was set to $60 \%$, considering the PC transmission $(90 \%)$ and ABS absorption efficiency (70\%). The input heat source was assumed to be a 3D ellipsoidal Gaussian distribution with a skin depth in z-direction, as described as Equation (1).
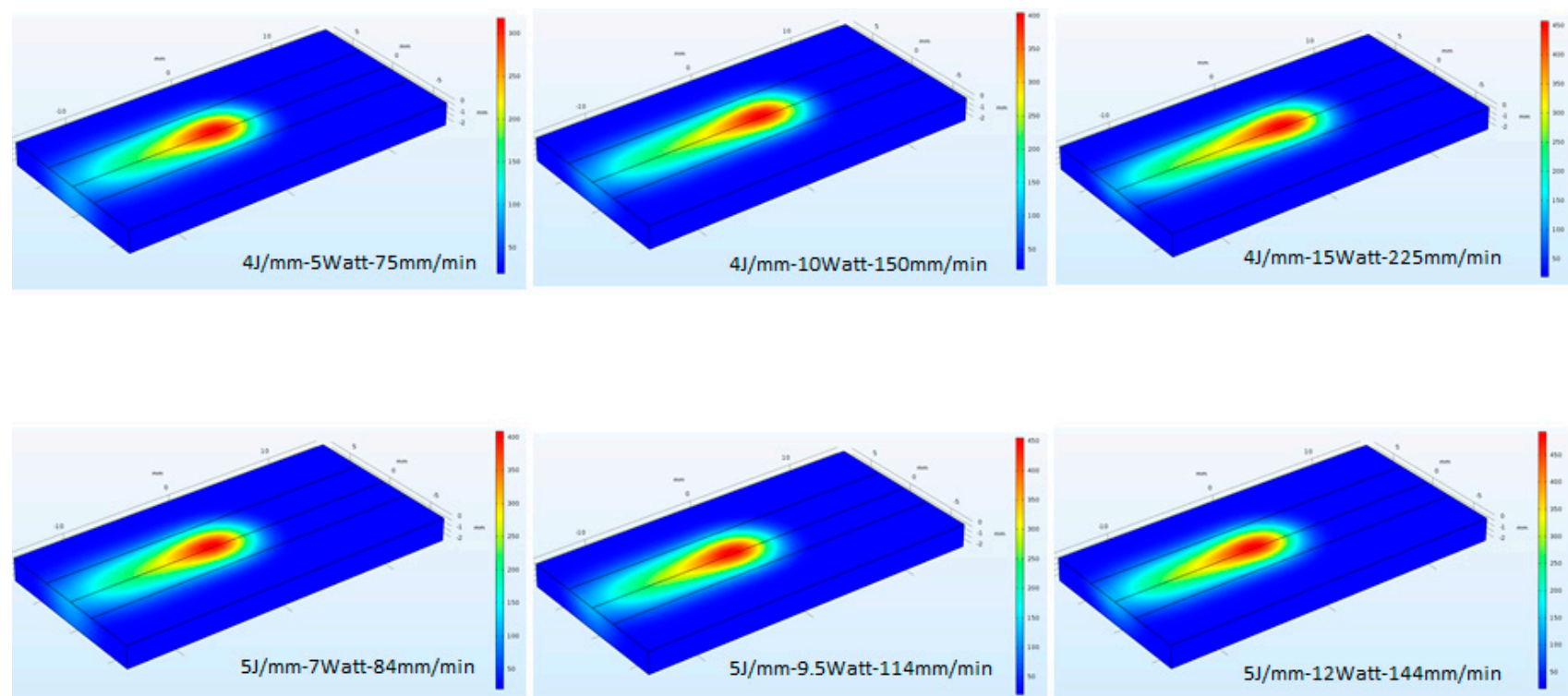

Figure 7. Simulation results for Table 1 (max. temp $500^{\circ} \mathrm{C}$ ).

To quantitatively analyze the results, the temperature distribution throughout the sample was traced from $0.25 \mathrm{~mm}$ underneath the surface at a position offset by $2.5 \mathrm{~mm}$ from the direction of travel of the laser (Figure 8). As shown in Figure 8, we predicted good results under the 4 and $5 \mathrm{~J} / \mathrm{mm}$ conditions, where the temperature was observed to vary from 120 to $180^{\circ} \mathrm{C}$ in all sections. However, as shown in Figure 8c, under low-power 
and low-speed conditions (experiment \#1-1), 3 Watt-60 mm/min, a lack of fusion was found as the maximum temperature was $100{ }^{\circ} \mathrm{C}$. At high power and high-speed transfer (experimental condition \#3-5), 17 Watt-204 mm/ min, the probability of welding failure was predicted to be high, as the maximum temperature would be at least $190^{\circ} \mathrm{C}$.

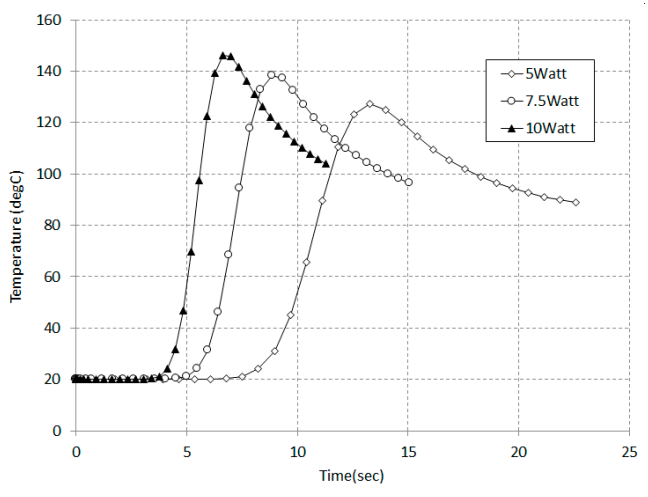

(a) $\mathrm{E}=3 \mathrm{~J} / \mathrm{mm}$.

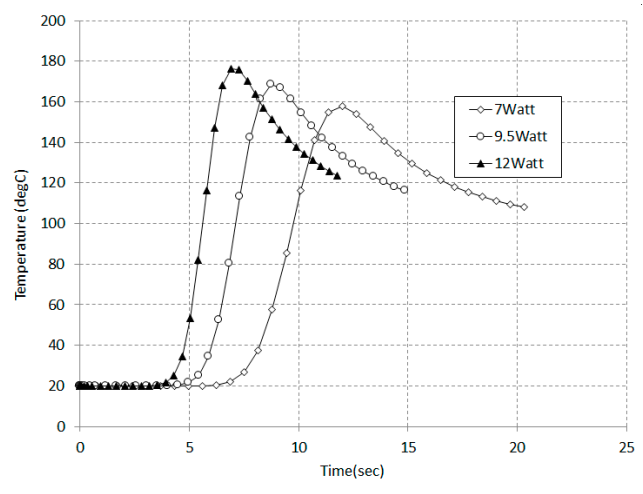

(b) $\mathrm{E}=5 \mathrm{~J} / \mathrm{mm}$.

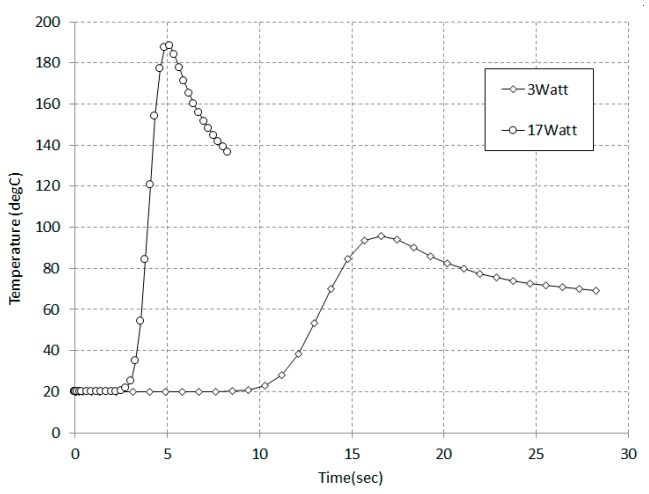

(c) Failed weld case.

Figure 8. Temperature distribution for different heat inputs.

\subsection{Deep Learning Algorithm and Process}

Welding quality was evaluated under each condition using the DL algorithm. Figure 9a-c show cases with defects and those with a good end-product along with the experimental schedule described in Table 1 . The welding quality was assessed based on the stochastic results, however the general trend of weld quality was identical to that of visual inspection. The detailed analysis is described in the following.

Under the low-energy condition where the heat input was set to $3 \mathrm{~J} / \mathrm{mm}$ (experiments $\# 1-1 \sim 1-5)$, the welding quality was not identical between the laser powers of 3 and $5.5 \mathrm{~W}$. To obtain quantitative results, DL was performed on photographs of the weld surface. The results in each case $(3,5.5,8,10.5$, and $13 \mathrm{~W})$ were classified as fail or good, with the accuracy of $>97 \%$ and $>99 \%$, respectively.

Under the mid-energy condition where the heat input was set to $4 \mathrm{~J} / \mathrm{mm}$ (experiments \#2-1 2-5), the welding quality was more acceptable than at lower energies regardless of the power levels. The laser power was increased from 5 to $15 \mathrm{~W}$ in increments of $2.5 \mathrm{~W}$, and the quality of the laser welding was evaluated based on the images, which were classified as good or fail using our DL algorithm with an accuracy $>99 \%$ or $>94 \%$, respectively. 

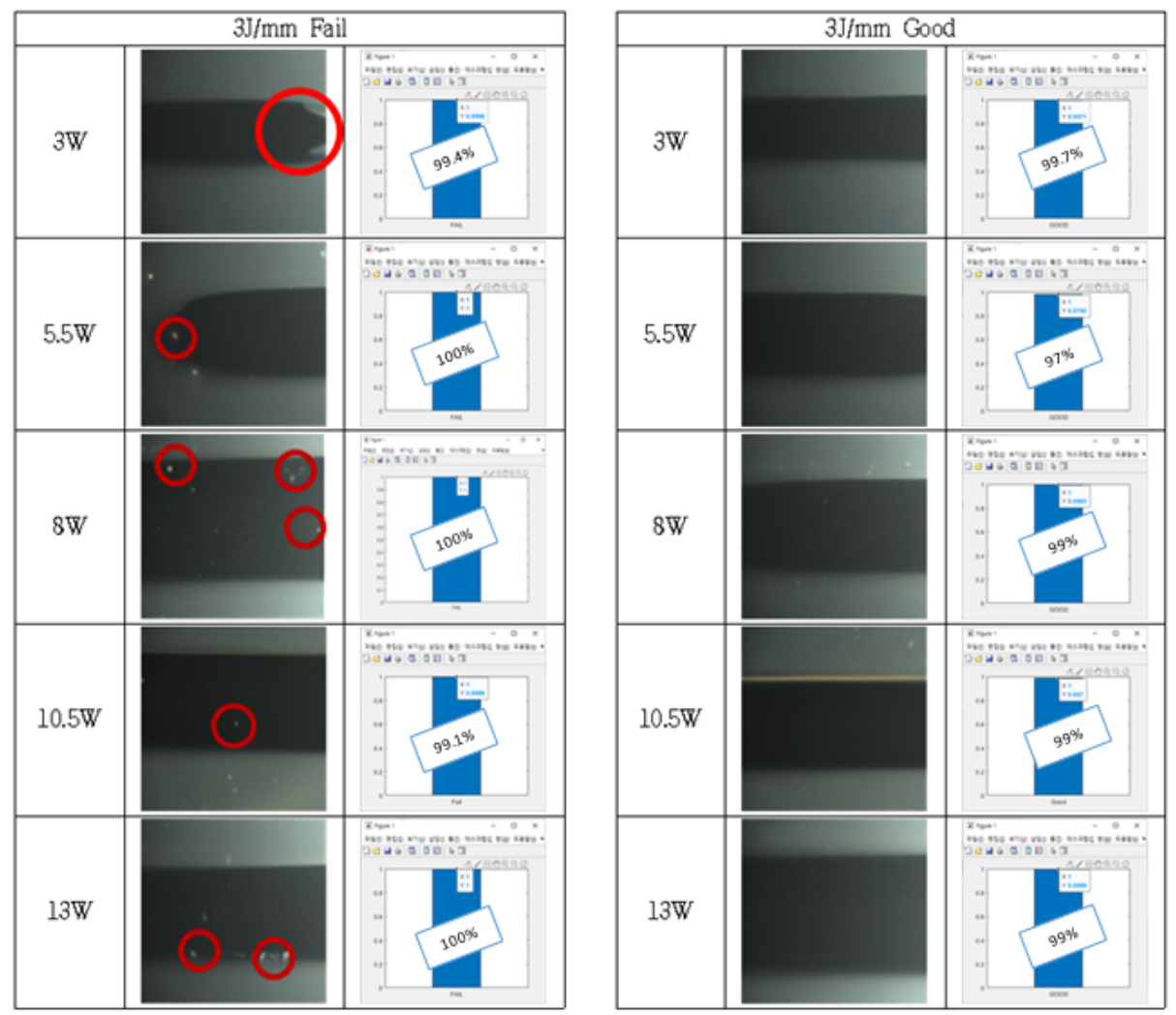

(a) Classification for $3 \mathrm{~J} / \mathrm{mm}$.
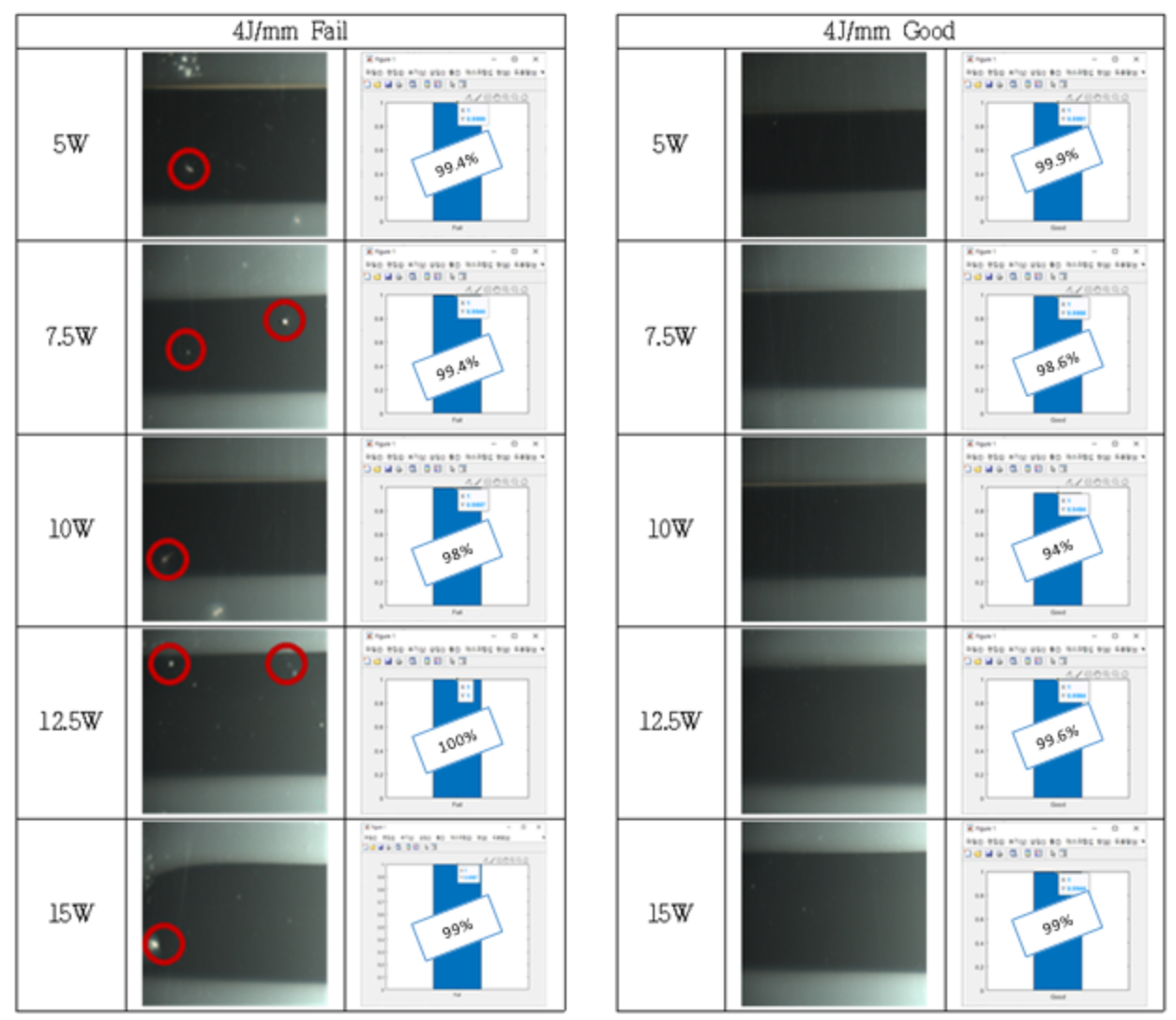

(b) Classification for $4 \mathrm{~J} / \mathrm{mm}$.

Figure 9. Cont. 

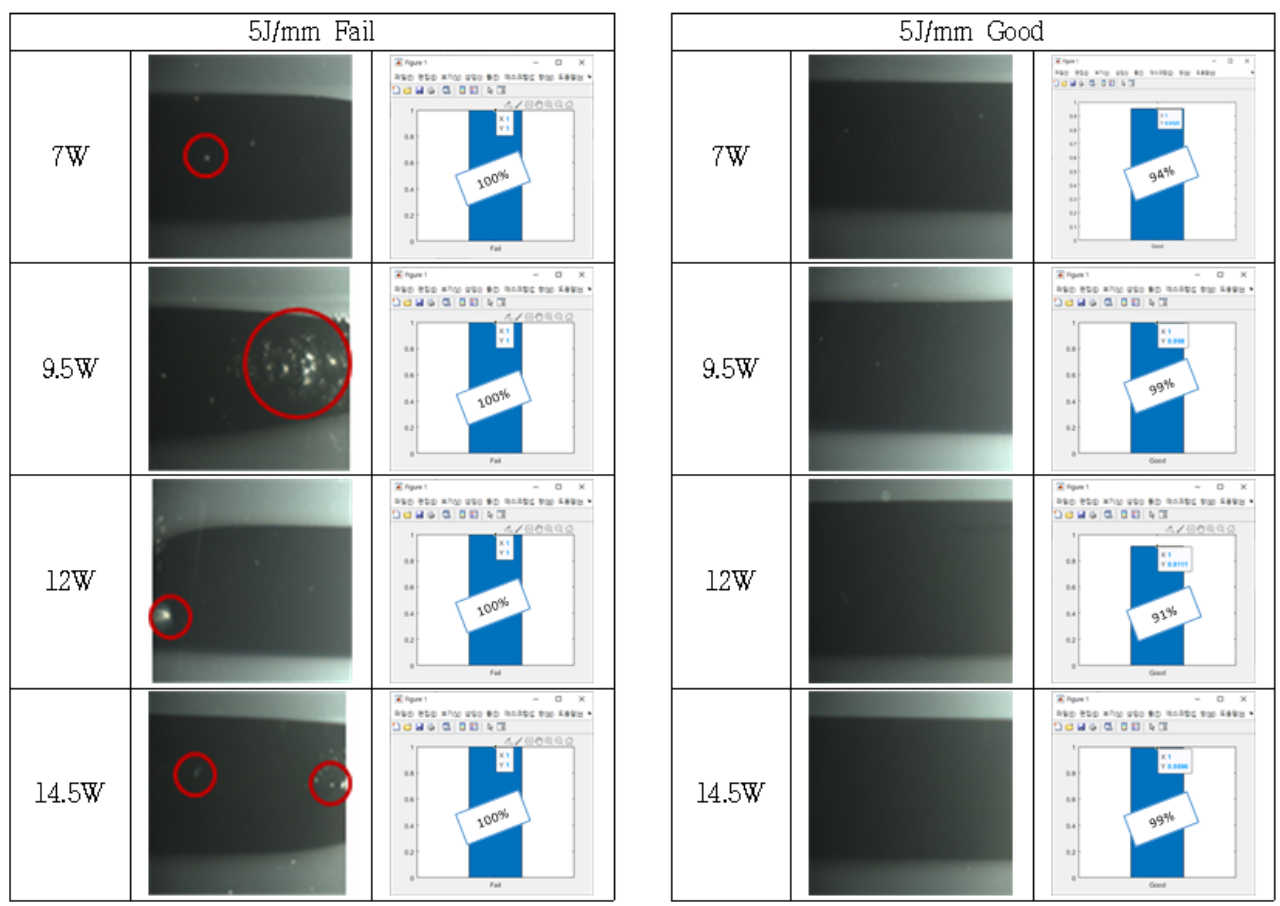

(c) Classification for $5 \mathrm{~J} / \mathrm{mm}$.

Figure 9. Quality evaluation by deep learning.

Under the high-energy condition where the heat input was set to $5 \mathrm{~J} / \mathrm{mm}$ (experiments \#3-1 3-5), the laser power was increased from 7 to $14.5 \mathrm{~W}$ in increments of $2.5 \mathrm{~W}$. The welding quality was acceptable except for experiments \#3-4 and \#3-5, where extremely high peak powers generated bubbles on the welded parts. Good and fail corresponded to accuracies of over $99 \%$ and $91 \%$, respectively.

As a summary of the DL results, the weld quality was statistically assessed, as shown in the bubble chart in Figure 10. While deterministically concluded in Figure 6, the results can be statistically estimated with a varying reliability. By providing statistical data to operators, one can select the best region for the welding where a high level of success can be achieved.

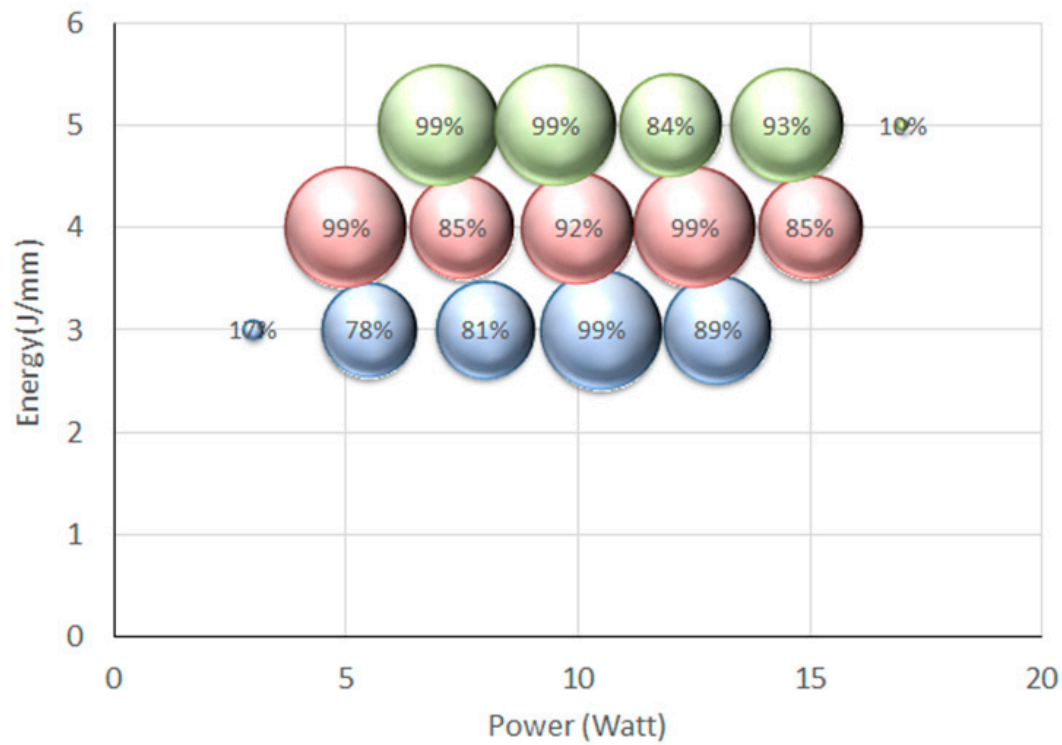

Figure 10. Stochastic prediction of weld quality by the developed Deep Learning 


\section{Conclusions and Discussion}

This paper reports an assessment of laser polymer joining by comparing visual inspection, computer simulation, and DL-based prediction. Based on the results of the preliminary research, these three modalities were used to increase the accuracy of the laser welding quality assessments.

For the experiments, energies in the range of $3 \sim 5 \mathrm{~J} / \mathrm{mm}$ were used. A total of 15 welding experiments were performed, and the laser power and welding speed were varied. Weld defects due to a lack of fusion were detected in some regions of specimens treated with the low-power laser $(3 \mathrm{~J} / \mathrm{mm})$, where a lack of fusion occurred due to underheating. Bubble-shaped weld defects were observed in some specimens treated with the high-power laser $(5 \mathrm{~J} / \mathrm{mm})$; melting occurred due to the overheating of the specimen.

Computer simulations were used to trace the boundaries of the fusion zone and yielded results similar to the visual inspection. In the lower-energy region, the energy may not be sufficient to fuse the specimen, whereas the high-energy region may have sufficient energy to break down the polymer chains.

Our results were then reevaluated using a novel DL algorithm which was modified from the AlexNet algorithm. Using a data acquisition (DAQ) system with a CCD camera, image data were acquired every $100 \mathrm{~ms}$ with $10 \mathrm{~ms}$ of exposure time, and approximately 1700 1900 samples were collected for each condition, with 28,500 images in total were used for training. The pre-trained quality evaluation indicated a highly reliable $(>98 \%)$ welding classification (fail or good). Specifically, the accuracy of the fail classification was over $99 \%$, and that of the good classification was greater than $97 \%$ in the $3 \mathrm{~J} / \mathrm{mm}$ condition. The accuracy was similar between the 4 and $5 \mathrm{~J} / \mathrm{mm}$ conditions. Failures were mainly due to pores or bubbles at the joining boundary. However, in some cases light reflected by scratches on the surface led to an incorrect classification as joining failure, which reduced the reliability of our DL algorithm-based quality evaluation.

According to the results of this study, welding quality assessments based on visual inspection, computer simulation, and DL-based inspection yield similar results. In conclusion, a visual inspection can be replaced with image-based DL and computer simulation for similarly reliable quality inspections but with significant cost and labor savings.

Author Contributions: Conceptualization, C.M.H., T.W.K., and H.W.C.; experiment, C.M.H.; deep learning, T.W.K.; formal analysis, H.W.C.; data curation, T.W.K.; writing-original draft preparation, C.M.H.; writing-review and editing, T.W.K.; supervision, H.W.C. All authors have read and agreed to the published version of the manuscript.

Funding: This research was funded by the Korea Nation Research Foundation, grant no.: NRF2019R1F1A1062594.

Institutional Review Board Statement: Not applicable.

Informed Consent Statement: Not applicable.

Data Availability Statement: Data sharing is not applicable to this article.

Acknowledgments: The English in this document has been checked by at least two professional editors, both native speakers of English. For a certificate, please see: http:/ / www.textcheck.com/ certificate/4rpc85.

Conflicts of Interest: The authors declare no conflict of interest. The funders had no role in the design of the study; in the collection, analysis, or interpretation of data; in the writing of the manuscript; or in the decision to publish the results.

\section{References}

1. Pelsmaeker, J.; Graulus, G.; Vlierberghe, S.; Thienpont, H.; Hemelrijckd, P.; Dubruel, D.; Ottevaere, H. Clear to clear laser welding for joining thermoplastic polymers: A comparative study based on physicochemical characterization. J. Mater. Process. Technol. 2018, 255, 808-815. [CrossRef]

2. Schricker, K.; Samfa, L.; Grätzel, M.; Ecke, G.; Bergman, J. Bonding mechanisms in laser-assisted joining of metal-polymer composites. J. Adv. Join. Process. 2020, 1, 100008. [CrossRef] 
3. Troschitz, J.; Vorderbrüggen, J.; Kupfer, R.; Gude, M.; Meschut, G. Joining of Thermoplastic Composites with Metals Using Resistance Element Welding. Appl. Sci. 2020, 10, 7251. [CrossRef]

4. Liu, F.; Dong, P.; Pei, X. A high-speed metal-to-polymer direct joining technique and underlying bonding mechanisms. J. Mater. Process. Technol. 2020, 280, 116610. [CrossRef]

5. Rodts, T.; Schmid, S.; Selles, M.; Pasang, T.; Sanchez-Caballero, S. Selective laser fiber welding on woven polymer fabrics for biomedical applications. Mater. Sci. Eng. 2019, C94, 628-634. [CrossRef] [PubMed]

6. Mingareev, I.; Weirauch, F.; Olowinsky, A.; Shah, L.; Kadwani, P.; Richardson, M. Welding of polymers using a 2um thulium fiber laser. Opt. Laser Technol. 2012, 44, 2095-2099.

7. Frick, T.; Schkutow, S. Laser transmission welding of polymers-Irradiation strategies for strongly scattering materials. $L A N E$ 2018, 74, 538-543. [CrossRef]

8. Matsumoto, R. Indentation plastic joining of steel rod and polycarbonate plate. J. Mater. Process. Technol. 2020, $283,116719$.

9. Yang, H.; Li, Z.; Wang, S. The Analytical Prediction of Thermal Distribution and Defect Generation of Inconel 718 by Selective Laser Melting. Appl. Sci. 2020, 10, 7300. [CrossRef]

10. Yang, Y.; Yang, R.; Pan, L.; Ma, J.; Zhu, Y.; Diao, T.; Zhang, L. A lightweight deep learning algorithm for inspection of laser welding defects on safety vent of power battery. Comput. Ind. 2020, 123, 103306. [CrossRef]

11. Oh, S.; Ki, H. Deep learning model for predicting hardness distribution in laser heat treatment of AISI H13 tool steel. Appl. Therm. Eng. 2019, 153, 583-595.

12. Zhang, Y.; You, D.; Gao, X.; Zhang, N.; Gao, P. Welding defects detection based on deep learning with multiple optical sensors during disk laser welding of thick plates. J. Manuf. Syst. 2019, 51, 87-94. [CrossRef]

13. Zhang, Z.; Li, B.; Zhang, W.; Lu, R.; Wada, S.; Zhang, Y. Real-time penetration state monitoring using convolutional neural network for laser welding of tailor rolled blanks. J. Manuf. Syst. 2020, 54, 348-360. [CrossRef]

14. Zhang, B.; Hong, K.; Shin, Y. Deep-learning-based porosity monitoring of laser welding process. Manuf. Lett. 2019, 23, 62-66. [CrossRef]

15. Tripathi, P.; Bag, S. CNN-DMRI: A Convolutional Neural Network for Denoising of Magnetic Resonance Images. Pattern Recognit. Lett. 2019, 135, 57-63. [CrossRef]

16. Jeong, E.-R.; Lee, E.-S.; Joung, J.; Oh, H. Convolutional Neural Network (CNN)-Based Frame Synchronization Method. Appl. Sci. 2020, 10, 7267. [CrossRef]

17. Kim, J.; Lee, J.-K. Stochastic Detection of Interior Design Styles Using a Deep-Learning Model for Reference Images. Appl. Sci. 2020, 10, 7299. [CrossRef]

18. Gunther, J.; Pilarski, P.; Helfrich, G.; Shen, H.; Diepold, K. First steps towards an intelligent laser welding architecture using deep neural networks and reinforcement learning. Procedia Technol. 2014, 15, 474-483. [CrossRef]

19. Acrylonitrile Butadiene Styrene (ABS) Typical Properties Generic ABS. 2020. Available online: https://www.ulprospector.com (accessed on 1 November 2020). 